\title{
Preconditioning Methods for Linear Systems with Saddle Point Matrices
}

\author{
Owe Axelsson ${ }^{1}$ and Maya Neytcheva ${ }^{2}$ \\ 1 Department of Mathematics, University of Nijmegen, Toernooiveld 1 \\ 6525 ED Nijmegen, The Netherlands, axelsson@sci.kun.nl \\ 2 Department of Scientific Computing, Uppsala University \\ Box 120, 75104 Uppsala, Sweden, maya@tdb.uu.se
}

\begin{abstract}
Preconditioning methods for matrices on saddle point form, as typically arising in constrained optimization problems, are surveyed. Special consideration is given to two methods: a nearly symmetric block incomplete factorization preconditioning method and an indefinite matrix preconditioner. Both methods result in eigenvalues with positive real parts and small or zero imaginary parts. The behaviour of the methods are illustrated on solving a regularized Stokes problem....
\end{abstract}

\section{Introduction}

Constrained optimization problems involve at least two variables, one of which is the so-called Lagrange multiplier. In the context of mixed variable or constrained finite element methods, the Lagrange multiplier acts as a natural physical variable. The arising systems are normally symmetric and indefinite, but have a block matrix structure which can be utilized. Various types of preconditioners for such systems have been proposed through the years. They include block diagonal preconditioners and block incomplete factorization preconditioners where the iteration matrix is symmetrizable but still indefinite, requiring some generalized iterative solution algorithm such as a minimum residual conjugate gradient method. Reduced matrix approaches, where a Schur complement system for one of the variables is solved, have also been proposed. The latter system is normally symmetric and positive definite and a symmetric positive definite preconditioner can be chosen. If one uses inner iterations, perturbations occur so one may be forced to use a (truncated) version of a generalized conjugate gradient method, such as GCG or GMRES.

Finally, indefinite preconditioners have been proposed. For the simplest type of those, the corresponding preconditioned matrix has only positive eigenvalues but has, in general, a deficient eigenvector space, i.e., the set of eigenvectors do not span the whole space. This may cause problems in the convergence of the iterative solution method.

In this paper, the above methods are surveyed. Two methods are given particular attention and it is shown how to avoid the eigenvector deficiency problem referred to above. An extension of this approach to a regularized system is 
presented. Regularization is needed for systems not satisfying the so called LBBstability condition. The regularization corresponds to the use of an augmented Lagrangian method in constrained optimization.

The remainder of the paper contains the following parts. Section 2 presents preconditioning methods for matrices on (regularized) saddle point form. Section 3 contains some numerical tests for the regularized Stokes solver in 2D.

\section{Solving indefinite matrix problems by iteration; preconditioners for indefinite matrix problems}

We consider here indefinite matrices of the form

$$
\mathcal{A}=\left[\begin{array}{cc}
M & B^{T} \\
B & 0
\end{array}\right] \text { and linear systems } \quad\left[\begin{array}{cc}
M & B^{T} \\
B & 0
\end{array}\right]\left[\begin{array}{l}
\mathbf{u} \\
\mathbf{x}
\end{array}\right]=\left[\begin{array}{l}
\mathbf{a} \\
\mathbf{b}
\end{array}\right],
$$

where $B$ has full row $\operatorname{rank}(=m)$ and $M$, of order $(n \times n)$, is symmetric. The block matrix $M$ can be indefinite in general, but we assume that $M$ is positive definite on $\operatorname{ker}(B)$, which implies that $\varepsilon M+B^{T} B, 0<\varepsilon<\varepsilon_{0}$, is positive definite for some sufficiently small $\varepsilon_{0}$. Such problems occur in equality constrained optimization problems, for instance.

If $M$ is indefinite, we may replace the system in (1) by

$$
\left[\begin{array}{cc}
M+\frac{1}{\varepsilon} B^{T} B & B^{T} \\
B & 0
\end{array}\right]\left[\begin{array}{l}
\mathbf{u} \\
\mathbf{x}
\end{array}\right]=\left[\begin{array}{c}
\mathbf{a}+\frac{1}{\varepsilon} B^{T} \mathbf{b} \\
\mathbf{b}
\end{array}\right],
$$

which is equivalent to the latter in the sense that both systems have the same solution. In particular, since $M+\frac{1}{\varepsilon} B^{T} B$ is positive definite, it follows readily that a solution exists. Due to the above equivalence we can equally well assume that $M$ in (1) is positive definite from the onset.

Solving indefinite matrix problems by iteration requires special care, because the iterative method may diverge, or a breakdown (division by zero), or a near breakdown (division by a number which is small) can occur. As is well-known, division by small numbers usually causes large relative round-off errors.

We shall now consider various approaches to solve (1).

\subsection{Schur complement approaches}

One way to solve (1) is via the Schur complement $S=-B M^{-1} B^{T}$. We then eliminate the first component $(\mathbf{u})$ to get

$$
B M^{-1} B^{T} \mathbf{x}=B M^{-1} \mathbf{a}-\mathbf{b},
$$

which is solved first, followed by $M \mathbf{u}=\mathbf{a}-B^{T} \mathbf{x}$. Here $-S$ is symmetric and also positive definite since $B$ has full rank. Clearly, in an iterative method there is no need to form the matrix $S$ explicitly, as it is only the action of the matrix which is needed. In each iteration step one such action is performed. However, each action 
requires the solution of the "inner" system with the matrix $M$ which, in general, for large scale problems is solved by iteration as well. Hence, a coupled innerouter iteration method must be used. The major expense in such an iterative scheme is normally related to the actions of $M^{-1}$, and the accuracy with which the systems with $M$ are solved plays thereby an important role.

When $M$ and/or $B M^{-1} B^{T}$ are ill-conditioned, it can be efficient to use the corresponding reformulation of the problem in (2). There holds (see e.g. [1]) the following lemma.

Lemma 1. Let $M_{\varepsilon}=\varepsilon M+B^{T} B$, where $M$ is positive definite and $B$ has full rank. Then

$B M_{\varepsilon}^{-1}=\left(\varepsilon I+B M^{-1} B^{T}\right) B M^{-1}, B M_{\varepsilon}^{-1} B^{T}=I-\varepsilon\left(\varepsilon I+B M^{-1} B^{T}\right)^{-1}$, and the eigenvalues of $B M_{\varepsilon}^{-1} B^{T}$ are contained in the interval $\left[\frac{\lambda_{1}}{\varepsilon+\lambda_{1}}, 1\right)$, where $\lambda_{1}$ is the smallest eigenvalue of $B M^{-1} B^{T}$.

Lemma 1 shows that, under the stated assumptions, we can make the matrix $B M_{\varepsilon}^{-1} B^{T}$ arbitrarily well-conditioned by choosing $\varepsilon$ sufficiently small. Hence, we can get an arbitrarily fast rate of convergence, leading to just a few iteration steps, when solving the reduced system of (2), i.e.,

$$
B M_{\varepsilon}^{-1} B^{T} \mathbf{x}=B M_{\varepsilon}^{-1}\left(\mathbf{a}+\frac{1}{\varepsilon} B^{T} \mathbf{b}\right)-\frac{1}{\varepsilon} \mathbf{b},
$$

by an iterative solution method such as the conjugate gradient (CG) method, with no need to use a preconditioning.

Remark 1. There is an alternative derivation of (2). The system (2) is namely the saddle point matrix for the augmented Lagrangian for the optimization problem $\min \left\{\frac{1}{2} \mathbf{u}^{T} M \mathbf{u}-\mathbf{a}^{T} \mathbf{u}\right\}$, subject to the constraint $B \mathbf{u}=\mathbf{b}$. If $\mathbf{x}$ is the Lagrangian multiplier, the augmented Lagrangian takes the form $\inf _{\mathbf{x}} \sup \left\{\frac{1}{2} \mathbf{u}^{T} M \mathbf{u}-\mathbf{a}^{T} \mathbf{u}+\mathbf{x}^{T}(B \mathbf{u}-\mathbf{b})+\frac{1}{2 \varepsilon}(B \mathbf{u}-\mathbf{b})^{T}(B \mathbf{u}-\mathbf{b})\right\}$, where $\varepsilon>0$. The idea with the augmented Lagrangian is to improve the conditioning of the matrix $M$ or, if $M$ is indefinite, let $\varepsilon$ be sufficiently small so that $M+\frac{1}{\varepsilon} B^{T} B$ is positive definite.

As we have seen, the reduced system gets increasingly well-conditioned by choosing $\varepsilon$ smaller. On the other hand, as $\varepsilon$ decreases, $M_{\varepsilon}=\varepsilon M+B^{T} B$ becomes increasingly ill-conditioned, which may require more computational efforts for solving the inner systems with $M_{\varepsilon}$. The optimal choice of $\varepsilon$ for smallest total computational complexity must be be a balance between the rate of convergence of the global method to solve (4) and the cost of solving systems with $M_{\varepsilon}$.

Using the relations in Lemma 1 an elementary computation shows that (4) can be rewritten in the form

$$
\mathbf{u}-\varepsilon\left(\varepsilon I+B M^{-1} B^{T}\right)^{-1}\left(\mathbf{u}+\frac{1}{\varepsilon}\left(B M^{-1} \mathbf{a}-\mathbf{b}\right)\right)=\mathbf{0},
$$

which can be used when computing the residuals in the iterative (CG) method. 
Assuming that $M^{-1}$ a is computed initially before the iterations, the latter method requires the action of the matrix $\varepsilon I+B M^{-1} B^{T}$, which can be somewhat better conditioned for a proper choice of $\varepsilon$ than $B M^{-1} B^{T}$, when $\lambda_{1}$ is small, as its smallest eigenvalue is $\lambda_{1}+\varepsilon$ while its largest eigenvalue is negligibly increased. Each action of this matrix requires essentially the same amount of computation as the action of $B M^{-1} B^{T}$.

\subsection{Block Gauss-Seidel preconditioners}

We consider now preconditioners for the whole, unreduced, system (1).

A simple, but still efficient preconditioner has the form $\mathcal{A}_{1}=\left[\begin{array}{cc}D_{1} & 0 \\ B & -D_{2}\end{array}\right]$, where $D_{1}$ and $D_{2}$ are symmetric positive definite preconditioners to $M$ and $B D_{1}^{-1} B^{T}$, respectively. An elementary computation reveals that the preconditioned matrix is

$$
\left[\begin{array}{cc}
D_{1} & 0 \\
B & -D_{2}
\end{array}\right]^{-1}\left[\begin{array}{cc}
M & B^{T} \\
B & 0
\end{array}\right]=\left[\begin{array}{cc}
I_{1} & D_{1}^{-1} B^{T} \\
0 & D_{2}^{-1} B D_{1}^{-1} B^{T}
\end{array}\right]+\left[\begin{array}{cc}
D_{1}^{-1}\left(M-D_{1}\right) & 0 \\
D_{2}^{-1} B D_{1}^{-1}\left(M-D_{1}\right) & 0
\end{array}\right] .
$$

It follows that if $D_{1}=M$ then the eigenvalues of the preconditioned matrix are real and equal to the unit number (with multiplicity at least $n$ ) and the eigenvalues of $D_{2}^{-1} B D_{1}^{-1} B^{T}$, respectively, which latter are positive. By choosing $D_{2}$ sufficiently close to $B D_{1}^{-1} B^{T}$ we can hence cluster the eigenvalues around the unit number.

If $D_{1}$ is not equal to $M$ we get complex eigenvalues in general, but if $D_{1}$ is sufficiently close to $M$ the eigenvalues have positive real parts, close to the unit number and small imaginary parts.

The arising preconditioned system can be solved with a minimal residual iterative method. Depending on the expense in solving systems with $D_{1}$ and $D_{2}$, this method can work quite efficiently.

Remark 2. Preconditioners of the above form are also suitable for cases when $M$ itself is nonsymmetric, as for the linearized Navier-Stokes equations, see e.g. [9, $7,8]$. As pointed out in [8], if $D_{2}$ takes the form $D_{2}=\left(B B^{T}\right)\left(B M B^{T}\right)^{-1}\left(B B^{T}\right)$, then under a certain additional condition, all eigenvalues of $\mathcal{A}^{-1} \mathcal{A}$ equal the unit number and no Jordan block has order higher than two.

Preconditioners of the form $\left(B B^{T}\right)\left(B M B^{T}\right)^{-1}\left(B B^{T}\right)$ to $B M^{-1} B^{T}$ have been considered previously in [4] for nonlinear diffusion problems, where it enabled for a cheap update of the nonlinear matrix $M$.

\subsection{Congruence transformations}

In order to avoid the strongly unsymmetric matrix in the former method, we can use a symmetric form of the preconditioning matrix. Consider first

$$
\tilde{\mathcal{A}}=\left[\begin{array}{cc}
L^{-1} & 0 \\
0 & N^{-1}
\end{array}\right]\left[\begin{array}{cc}
M & B^{T} \\
B & 0
\end{array}\right]\left[\begin{array}{cc}
L^{-T} & 0 \\
0 & N^{-T}
\end{array}\right]=\left[\begin{array}{cc}
L^{-1} M L^{-T} & L^{-1} B^{T} N^{-T} \\
N^{-1} B L^{-T} & 0
\end{array}\right],
$$


where $L, N$ are nonsingular matrices, the choice of which will be discussed below. The negative Schur complement of $\widetilde{\mathcal{A}}$ equals $N^{-1} B M^{-1} B^{T} N^{-T}$, which does not depend on $L$. Therefore, we can choose $N$ independently of $L$, so that the outer matrix $N^{-1}\left(B M^{-1} B^{T}\right) N^{-1}$ is well-conditioned. By choosing $L L^{T}$ as an accurate preconditioner of $M$, such as an incomplete Cholesky factorization, we can solve the arising inner systems with $L^{-1} M L^{-T}$ efficiently.

The matrix $\widetilde{\mathcal{A}}$ in (6) is still on saddle point form. The next congruence transformation reduces $\widetilde{\mathcal{A}}$ to block-diagonal form. Consider then first the more general matrix $A=\left[\begin{array}{ll}A_{11} & A_{12} \\ A_{21} & A_{22}\end{array}\right]$, where $A_{11}$ is symmetric positive definite (s.p.d.), $A_{21}^{T}=A_{12}$ and $A_{22}$ is symmetric. Then, if $S=A_{22}-A_{21} A_{11}^{-1} A_{12}$,

$$
\left[\begin{array}{cc}
I_{1} & 0 \\
-A_{21} A_{11}^{-1} & I_{2}
\end{array}\right]\left[\begin{array}{ll}
A_{11} & A_{12} \\
A_{21} & A_{22}
\end{array}\right]\left[\begin{array}{cc}
I_{1}-A_{11}^{-1} A_{12} \\
0 & I_{2}
\end{array}\right]=\left[\begin{array}{cc}
A_{11} & 0 \\
0 & S
\end{array}\right]
$$

Hence, this transformation reduces $A$ to a block-diagonal form with symmetric block matrices. For a saddle point matrix, where $A_{22}=0$, we have $S=$ $-A_{21} A_{11}^{-1} A_{12}$, so $S$ is negative (semi)definite.

In this method one must find a preconditioner to $S$. In some problems such as arising in certain partial differential equations like the Stokes problem, $S$ may be quite well-conditioned and it may therefore suffice to precondition it by a diagonal matrix. However, systems with $A_{11}$ must still be solved.

We consider next applying a transformation to a matrix with positive definite symmetric part, where we have changed the sign in the lower block rows in one of the factors (as in [3] among others). We can apply the alternative transformation

$$
\left[\begin{array}{cc}
I_{1} & 0 \\
A_{21} A_{11}^{-1} & -I_{2}
\end{array}\right]\left[\begin{array}{ll}
A_{11} & A_{12} \\
A_{21} & A_{22}
\end{array}\right]\left[\begin{array}{cc}
I_{1}-A_{11}^{-1} A_{12} \\
0 & I_{2}
\end{array}\right]=\left[\begin{array}{cc}
A_{11} & 0 \\
0 & -S
\end{array}\right]
$$

in which case, for a saddle point problem, $-S$ is positive (semi)definite and all eigenvalues are nonnegative (positive if $-S$ is positive definite, which latter holds if $A_{21}$ has full rank).

When applying transformation (8) we must solve systems with $A_{11}$ and in the corresponding iterative method we must solve the Schur complement system, which again involves the action of $A_{11}^{-1}$. Clearly, such a method is not viable, as we might better have solved just the reduced system for the component $\mathbf{x}$ first and then computed the other component $(\mathbf{u})$. Therefore, when applied for the saddle point matrix (1), we combine the transformations in (7) and (8), i.e., we use the left and right transformations $\left[\begin{array}{cc}I_{1} & 0 \\ \widetilde{B} M^{-1} & -I_{2}\end{array}\right]\left[\begin{array}{cc}L^{-1} & 0 \\ 0 & N^{-1}\end{array}\right]$ and $\left[\begin{array}{cc}L^{-T} & 0 \\ 0 & N^{-T}\end{array}\right]\left[\begin{array}{cc}I_{1} & \widetilde{M}^{-1} \widetilde{B}^{T} \\ 0 & I_{2}\end{array}\right]$, where $\widetilde{M}=L^{-1} M L^{-T}$ and $\widetilde{B}=N^{-1} B L^{-T}$. The transformed matrix takes then the form

$$
\left[\begin{array}{cc}
L^{-1} & 0 \\
\widetilde{B} \widetilde{M}^{-1} L^{-1} & -N^{-1}
\end{array}\right]\left[\begin{array}{cc}
M & B^{T} \\
B & 0
\end{array}\right]\left[\begin{array}{cc}
L^{-T}-L^{-T} \widetilde{M}^{-1} \widetilde{B}^{T} \\
0 & N^{-T}
\end{array}\right]=\left[\begin{array}{cc}
\widetilde{M} & 0 \\
0 & \widetilde{B} \widetilde{M}^{-1} \widetilde{B}^{T}
\end{array}\right],
$$


which is block-diagonal. However, this matrix transformation still requires the action of $\widetilde{M}^{-1}=L^{T} M^{-1} L$. We replace therefore finally $\widetilde{M}$ with a diagonal matrix $\widetilde{D}$ and and elementary computation shows that the left and right preconditioned matrix takes then the form

$$
\begin{aligned}
& {\left[\begin{array}{cc}
L^{-1} & 0 \\
\widetilde{B} \widetilde{D}^{-1} L^{-1}-N^{-1}
\end{array}\right]\left[\begin{array}{cc}
M & B^{T} \\
B & 0
\end{array}\right]\left[\begin{array}{cc}
L^{-T}-L^{-T} \widetilde{D}^{-1} \widetilde{B}^{T} \\
0 & N^{-T}
\end{array}\right]=} \\
& {\left[\begin{array}{c}
\widetilde{M} \\
\left.\widetilde{B}\left(\widetilde{D}^{-1} \widetilde{M}-I_{1}\right) 2 \widetilde{B} \widetilde{D}^{-1} \widetilde{B}^{T}-\widetilde{M} \widetilde{D}^{-1}\right) \widetilde{B}^{-1} \widetilde{M} \widetilde{D}^{-1} \widetilde{B}^{T}
\end{array}\right]}
\end{aligned}
$$

This preconditioner involves actions of $L^{-1}, L^{-T}$ and $N^{-1}, N^{-T}$, as well as of $\widetilde{B} \widetilde{D}^{-1}$ and $\widetilde{D}^{-1} \widetilde{B}^{T}$, which latter involves additional actions of $L^{-1}$ and ${ }^{-T}$.

If $L L^{T}$ is a sufficiently close approximation to $M$, then $\widetilde{M}$ is nearly diagonal and we may choose $\widetilde{D}$ as a sufficiently close approximation of $\widetilde{M}$ and therefore the eigenvalues of the symmetric part of (9) will be positive. Furthermore, the skew-symmetric part gets arbitrarily small. Hence, the transformed matrix has eigenvalues with positive real part, which cluster around the unit number as the imaginary parts of the eigenvalues are small. Unfortunately, this preconditioner involves much computation - two actions of $L^{-1}$ and $L^{-T}$ and one action of $N^{-1}$ and $N^{-T}$.

The final preconditioner we present here involves less computational effort and gives positive eigenvalues as well, even though it is based on an indefinite matrix.

\subsection{A preconditioner on saddle point form}

We consider now finally preconditioners of the same, indefinite, form as the given matrix. For the analysis of the first preconditioner we shall use the next lemma.

Lemma 2. Let $B, C, E$ be real matrices of order $n \times m, m \times m$ and $n \times n$ respectively, where $B$ has full rank $(=m), C$ is positive definite and $E$ is symmetric. Then the eigenvalues of the generalized eigenvalue problem

$$
\gamma\left[\begin{array}{cc}
I & B^{T} \\
B & -C
\end{array}\right]\left[\begin{array}{l}
\mathbf{u} \\
\mathbf{x}
\end{array}\right]=\left[\begin{array}{cc}
E & 0 \\
0 & 0
\end{array}\right]\left[\begin{array}{l}
\mathbf{u} \\
\mathbf{x}
\end{array}\right], \quad|\mathbf{u}|+|\mathbf{x}| \neq \mathbf{0}
$$

where $\mathbf{u} \in \mathbb{C}^{n}$ and $\mathbf{x} \in \mathbb{C}^{m}$, satisfy

(a) $\gamma=\frac{\mathbf{u}^{*} E \mathbf{u}}{\mathbf{u}^{*}\left(I+B^{T} C^{-1} B\right) \mathbf{u}} \neq 0$, if $\quad E \mathbf{u} \neq \mathbf{0}$ and $\gamma=0$, if and only if $\mathbf{u}=\mathbf{0}, \mathbf{x} \neq$ 0,

(b) the dimension of the eigenvector space corresponding to the zero eigenvalue is $m+q$, where $q=\operatorname{dim}\{k e r(E)\}$;

(c) the nonzero eigenvalues are contained in the interval $\lambda_{\min }(E) \leq \gamma \leq \lambda_{\max }(E)$.

Consider now the generalized eigenvalue problem (10), where $C=0$. Here it holds $\gamma\left(\mathbf{u}+B^{T} \mathbf{x}\right)=E \mathbf{u}$ and $\gamma B \mathbf{u}=\mathbf{0}$. Thus, in this case, at least one of $\gamma=0$ 
or $B \mathbf{u}=\mathbf{0}$ must hold. If $B \mathbf{u}=\mathbf{0}$ but $\gamma \neq 0$ then $\gamma B B^{T} \mathbf{x}=B E \mathbf{u}$ (i.e., there holds here $E \mathbf{u} \neq \mathbf{0}$ ) and $\gamma \mathbf{u}^{*} \mathbf{u}=\mathbf{u}^{*} E \mathbf{u}$, i.e., $\mathbf{x}=\frac{1}{\gamma}\left(B B^{T}\right)^{-1} B E \mathbf{u}$, where $\gamma=$ $\frac{\mathbf{u}^{*} E \mathbf{u}}{\mathbf{u}^{*} \mathbf{u}}$. Further, $\gamma \mathbf{u}+B^{T}\left(B B^{T}\right)^{-1} B E \mathbf{u}=E \mathbf{u}$ or $\gamma \mathbf{u}=\left(I-B^{T}\left(B B^{T}\right)^{-1} B\right) E \mathbf{u}$. Hence, for any $\mathbf{u} \in \operatorname{ker}(B), \mathbf{u} \neq \mathbf{0}$ and eigenvalue $\gamma \neq 0$, there holds that $\left[\mathbf{u}, \frac{1}{\gamma}\left(B B^{T}\right)^{-1} B E \mathbf{u}\right]^{t}, \gamma=\mathbf{u}^{*} E \mathbf{u} / \mathbf{u}^{*} \mathbf{u}$ is an eigenvector for this $\gamma$.

If $\gamma=0$, then it must hold $E \mathbf{u}=\mathbf{0}$. If $E \mathbf{u}=\mathbf{0}$ then $\gamma=0$ is an eigenvalue for such a vector $\mathbf{u}$ and any $\mathbf{x}$. Hence, there holds that $\left[0, \mathbf{x}^{(i)}\right]^{T}, i=1,2, \cdots, m$ and $\left[\mathbf{u}^{(j)}, \mathbf{0}\right]^{T}, j=1,2, \cdots, q$ are eigenvectors for $\gamma=0$, where $\left\{\mathbf{x}^{(i)}\right\}_{1}^{m}$ span $\mathbb{R}^{m}$ and $E \mathbf{u}^{(j)}=\mathbf{0}, \mathbf{u}^{(j)} \neq \mathbf{0}$. Hence, there are $m+q$ linearly independent eigenvectors for $\gamma=0$.

For some of the eigenvectors $\mathbf{u}^{(j)}$ it may hold $\mathbf{u}^{(j)} \in \operatorname{ker}(B)$. If $\operatorname{ker}(E) \cap$ $\operatorname{ker}(B)=0$ then the algebraic multiplicity of $\gamma=0$ is $n+m-(n-m)=2 m$. In this case the index of eigenvector deficiency is $2 m-(m+q)=m-q$.

If, however, $\operatorname{ker}(E) \cap \operatorname{ker}(B) \neq 0$, then the algebraic multiplicity is increased and the index of eigenvector deficiency is correspondingly increased.

If one can construct $D$ so that $E=D^{\frac{1}{2}} M D^{-\frac{1}{2}}-I$ has $m$ zero eigenvalues, then there would be no eigenvector deficiency. It is clear, however, that this requirement is not viable for most applications.

Consider now the matrix $\mathcal{A}=\left[\begin{array}{cc}M & B^{T} \\ B & -C\end{array}\right]$. Let $D$ be a symmetric and positive definite preconditioner to $M$ and let $\left[\begin{array}{ll}D & B^{T} \\ B & -C\end{array}\right]$ be a preconditioner to $\mathcal{A}$. For the generalized eigenvalue problem $\lambda\left[\begin{array}{ll}D & B^{T} \\ B & -C\end{array}\right]\left[\begin{array}{l}\mathbf{u} \\ \mathbf{x}\end{array}\right]=\mathcal{A}\left[\begin{array}{l}\mathbf{u} \\ \mathbf{x}\end{array}\right]$ we have $\gamma\left[\begin{array}{ll}D & B^{T} \\ B & -C\end{array}\right]\left[\begin{array}{l}\mathbf{u} \\ \mathbf{x}\end{array}\right]=$ $\left[\begin{array}{rr}M-D & 0 \\ 0 & 0\end{array}\right]\left[\begin{array}{l}\mathbf{u} \\ \mathbf{x}\end{array}\right]$, where $\gamma=\lambda-1$, or $\gamma\left[\begin{array}{cc}I & \widetilde{B}^{T} \\ \widetilde{B} & -C\end{array}\right]\left[\begin{array}{l}\widetilde{\mathbf{u}} \\ \mathbf{x}\end{array}\right]=\left[\begin{array}{ll}\widetilde{E} & 0 \\ 0 & 0\end{array}\right]\left[\begin{array}{l}\widetilde{\mathbf{u}} \\ \mathbf{x}\end{array}\right]$, where $\widetilde{B}=B D^{-\frac{1}{2}}, \widetilde{E}=D^{-\frac{1}{2}} M D^{-\frac{1}{2}}-I$ and $\widetilde{\mathbf{u}}=D^{-\frac{1}{2}} \mathbf{u}$.

This problem has the same form as the generalized eigenvalue problem in Lemma 2. Hence, the previous analysis of the eigenvalues and eigenvectors is applicable.

In this paper we propose the following preconditioner on regularized form,

$$
\left[\begin{array}{cc}
D_{1} & B^{T} \\
B & -C
\end{array}\right]
$$

where $D_{1}$ is a preconditioner to $M$. The systems with this preconditioner will be solved via the Schur complement $C+B D_{1}^{-1} B^{T}$.

A preconditioner for the Schur complement system must be found. In applications such as for Stokes problem, where $D_{1}$ is a sufficiently accurate preconditioner to $M$, it can be chosen as a diagonal matrix $D_{2}$. The computational effort in solving systems with the preconditioner (using the conjugate gradient method) is therefore not big. Furthermore, as we have already commented on, the number of outer iterations if using a GCG method will be few.

Remark 3. Indefinite preconditioners for the saddle point problem (10) have been proposed previously in [3] and [10]. However, it was not pointed out that 
the eigenvector space for the unit eigenvalue, $\lambda=1$, is deficient in general. This was, however, done in [11]. The eigenvector deficiency may cause problems in the iterative solution algorithm. For instance, the rate of convergence of minimal residual iterative methods are based on the expansion of the initial residual using the eigenvectors as basis vectors. This approach is inapplicable when the space is deficient. Alternatively, see e.g. [5], one can use estimates, based on the Jordan canonical form but this shows also a possible long delay in convergence. Other preconditioners have been proposed in [11] where one may avoid the eigenvalue deficiency problem. As we have seen, there is no eigenvalue deficiency if one uses the regularized form of the preconditioner.

\subsection{An indefinite preconditioner on factorized form}

Following [3], let now the preconditioner be given on factorized form

$$
\mathcal{A}_{0}=\left[\begin{array}{cc}
D & B^{T} \\
B & -R
\end{array}\right]=\left[\begin{array}{ll}
D & 0 \\
B & L
\end{array}\right]\left[\begin{array}{cc}
I_{1} & D^{-1} B^{T} \\
0 & -L^{T}
\end{array}\right]
$$

where $R=L L^{T}-B D^{-1} B^{T}$. Here $D$ is a preconditioner to $M$ and $L L^{T}$ is a preconditioner to $C+B D^{-1} B^{T}$. We will use $\mathcal{A}_{0}$ as a preconditioner to $\mathcal{A}=$ $\left[\begin{array}{cc}M & B^{T} \\ B & -C\end{array}\right]$. Depending on the choice of $L$ and $D$, this preconditioner can be positive definite or indefinite.

For its analysis, we consider then the generalized eigenvalue problem $\lambda \mathcal{A}_{0}\left[\begin{array}{l}\mathbf{u} \\ \mathbf{x}\end{array}\right]=$ $\mathcal{A}\left[\begin{array}{l}\mathbf{u} \\ \mathbf{x}\end{array}\right]$ or $\gamma\left[\begin{array}{ll}D & B^{T} \\ B & -R\end{array}\right]\left[\begin{array}{l}\mathbf{u} \\ \mathbf{x}\end{array}\right]=\left[\begin{array}{cc}M-D & 0 \\ 0 & R-C\end{array}\right]\left[\begin{array}{l}\mathbf{u} \\ \mathbf{x}\end{array}\right]$, where $\gamma=\lambda-1$. We find then $\gamma\left[\begin{array}{l}\mathbf{u} \\ \mathbf{x}\end{array}\right]=\left[\begin{array}{cc}D^{-1}-D^{-1} B^{T} S^{-1} B D^{-1} D^{-1} B^{T} S^{-1} \\ S^{-1} B D^{-1} & -S^{-1}\end{array}\right]\left[\begin{array}{cc}M-D & 0 \\ 0 & R-C\end{array}\right]\left[\begin{array}{l}\mathbf{u} \\ \mathbf{x}\end{array}\right]$, where $S=R+B D^{-1} B^{T}=L L^{T}$, or

$$
\gamma\left[\begin{array}{c}
\widetilde{\mathbf{u}} \\
\widetilde{\mathbf{x}}
\end{array}\right]=\left[\begin{array}{cc}
I_{1}-\widetilde{B}^{T} \widetilde{B} & \widetilde{B}^{T} \\
\widetilde{B} & -I_{2}
\end{array}\right]\left[\begin{array}{cc}
\widetilde{M}-I_{1} & 0 \\
0 & \widetilde{R}-\widetilde{C}
\end{array}\right]\left[\begin{array}{l}
\widetilde{\mathbf{u}} \\
\widetilde{\mathbf{x}}
\end{array}\right]
$$

where $\widetilde{\mathbf{u}}=D^{\frac{1}{2}} \mathbf{u}, \widetilde{\mathbf{x}}=L^{T} \mathbf{x}, \widetilde{B}=L^{-1} B D^{\frac{1}{2}}, \widetilde{M}=D^{-\frac{1}{2}} M D^{-\frac{1}{2}}, \widetilde{R}=L^{-1} R L^{-T}=$ $I_{2}-\widetilde{B} \widetilde{B}^{T}, \widetilde{C}=L^{-1} C L^{-T}$.

To analyse this further we make first the assumption that $\widetilde{M} \geq I_{1}$ and $\widetilde{R} \geq \widetilde{C}$, i.e., $I_{2} \geq \widetilde{C}+\widetilde{B} \widetilde{B}^{T}$. This holds if $D$ and $L L^{T}$ are proper preconditioners to $M$ and $C+B D^{-1} B^{T}$, respectively.

It follows then from $(13)$, that if $\widehat{\mathbf{u}}=\left(\widetilde{M}-I_{1}\right)^{\frac{1}{2}} \widetilde{\mathbf{u}}$ and $\widehat{\mathbf{x}}=(\widetilde{R}-\widetilde{C})^{\frac{1}{2}} \widetilde{\mathbf{x}}$,

$$
\gamma\left[\begin{array}{l}
\widehat{\mathbf{u}} \\
\widehat{\mathbf{x}}
\end{array}\right]=\left[\begin{array}{cc}
\left(\widetilde{M}-I_{1}\right)^{\frac{1}{2}}\left(I_{1}-\widetilde{B}^{T} \widetilde{B}\right)\left(\widetilde{M}-I_{1}\right)^{\frac{1}{2}}\left(\widetilde{M}-I_{1}\right)^{\frac{1}{2}} \widetilde{B}^{T}(\widetilde{R}-\widetilde{C})^{\frac{1}{2}} \\
(\widetilde{R}-\widetilde{C})^{\frac{1}{2}} \widetilde{B}\left(\widetilde{M}-I_{1}\right)^{\frac{1}{2}} & -(\widetilde{R}-\widetilde{C})
\end{array}\right]\left[\begin{array}{l}
\widehat{\mathbf{u}} \\
\widehat{\mathbf{x}}
\end{array}\right] .
$$


This matrix is symmetric but indefinite. Its eigenvalues are hence real and the absolute values of them can be controlled by choosing sufficiently accurate preconditioners $D$ to $M$ and $L L^{T}$ to $C+B D^{-1} B^{T}$. In this way we may get $\gamma>-1$, so $\lambda=\gamma+1>0$. On the other hand, if $\widetilde{R} \leq \widetilde{C}$, then (13) can be transformed to

$$
\gamma\left[\begin{array}{l}
\widehat{\mathbf{u}} \\
\widehat{\mathbf{x}}
\end{array}\right]=\left[\begin{array}{cc}
\left(\widetilde{M}-I_{1}\right)^{\frac{1}{2}}\left(I_{1}-\widetilde{B}^{T} \widetilde{B}\right)\left(\widetilde{M}-I_{1}\right)^{\frac{1}{2}}-\left(\widetilde{M}-I_{1}\right)^{\frac{1}{2}} \widetilde{B}^{T}(\widetilde{C}-\widetilde{R})^{\frac{1}{2}} \\
(\widetilde{C}-\widetilde{R})^{\frac{1}{2}} \widetilde{B}\left(\widetilde{M}-I_{1}\right)^{\frac{1}{2}} & (\widetilde{C}-\widetilde{R})
\end{array}\right]\left[\begin{array}{l}
\widehat{\mathbf{u}} \\
\widehat{\mathbf{x}}
\end{array}\right],
$$

where now $\widehat{\mathbf{x}}=(\widetilde{C}-\widetilde{R})^{\frac{1}{2}} \mathbf{x}$. In this case, the preconditioned matrix, after transformation, is nonsymmetric, but with positive definite symmetric part. Hence the eigenvalues may be complex but there holds that $\operatorname{Re}(\lambda) \geq 1$. Further, as before, the eigenvalues cluster around the unit number when $D$ is sufficiently close to $M$ and $L L^{T}$ is sufficiently close to $C+B D^{-1} B^{T}$.

Finally, if $\widetilde{M}-I_{1}$ and/or $\widetilde{R}-\widetilde{C}$ are indefinite, then the eigenvalues may be complex but we can estimate the absolute value of $\gamma$ by simple norm inequalities and clustering around the unit number occurs as before.

\section{$3 \quad$ Numerical tests}

We choose to illustrate the behaviour of the proposed indefinite system preconditioners on the stationary Stokes problem, described in detail in [2].

$$
\begin{gathered}
-\Delta u+p_{x}=f_{1}(x, y) \text { in } \Omega, \\
-\Delta v+p_{y}=f_{2}(x, y) \text { in } \Omega, \\
u_{x}+v_{y}=0 \text { in } \Omega \\
\left.u(x, y)\right|_{\partial \Omega}=g_{1}(x, y),\left.\quad v(x, y)\right|_{\partial \Omega}=g_{2}(x, y) .
\end{gathered}
$$

Problem 1. Let $\Omega=(0,1)^{2}$. The functions $f_{1}$ and $f_{2}$, and the boundary conditions are computed so that the exact solution of (15) is $u(x, y)=x^{3}+x^{2}-2 x y+x$, $v(x, y)=-3 x^{2} y+y^{2}-2 x y-y$ and $p(x, y)=x^{2}+y^{2}$.

We use the regularized method described above, namely, we solve a system with a matrix $\mathcal{A}=\left[\begin{array}{cc}M & B^{T} \\ B & -\sigma C\end{array}\right]$, and the choice of the regularization parameter $\sigma$ is broadly discussed in [2].

Problem 1 is solved using the preconditioned GCG-MR method (cf. [1]) and a preconditioner $\widetilde{\mathcal{A}}$ to $\mathcal{A}$ of the form (11). $D_{1}$ in our experiments is the AMLIpreconditioner constructed for the diagonal blocks of $M$. During each GCG-MR iteration, systems with the preconditioner are solved via its Schur complement $S_{\tilde{\mathcal{A}}}=-\left(\sigma C+B \operatorname{AMLI}[M] B^{T}\right)$. Relative stopping criteria are used for both GCG-MR and for the CG methods. $\left\|\mathbf{r}^{(k)}\right\| /\left\|\mathbf{r}^{(0)}\right\|$ is checked to be less than $10^{-6}$ and $10^{-4}$, respectively. The results of this experiment are shown in Table 1 . The systems with $S_{\widetilde{\mathcal{A}}}$ can be solved by a unpreconditioned CG method since for the particular regularized formulation its condition number is independent of the discretization parameter $h$ (column 4 in Table 1). One can further improve the total complexity of the method by using a preconditioned CG to solve $S_{\tilde{\mathcal{A}}}$ (column 5 in Table 1).

The numerical tests are performed in Matlab. 
Table 1. Method (11), Problem 1

\begin{tabular}{|l|c|c|c|c|}
\hline$h$ & size $(\mathcal{A})$ & GCG-MR iter. & Aver. CG iter. & Aver. PCG iter. \\
\hline \hline 0.0667 & $\begin{array}{c}768 \\
(3 \times 256)\end{array}$ & 7 & 24 & 14 \\
\hline 0.0323 & $\begin{array}{c}3072 \\
(3 \times 1024)\end{array}$ & 7 & 25 & 16 \\
\hline 0.0159 & $\begin{array}{c}12288 \\
(3 \times 4096)\end{array}$ & 8 & 25 & 18 \\
\hline 0.0039 & $\begin{array}{c}196608 \\
(3 \times 65536)\end{array}$ & 8 & 25 & 18 \\
\hline
\end{tabular}

\section{References}

1. Axelsson O.: Iterative Solution Methods, Cambridge University Press, Cambridge, 1994.

2. Axelsson O., Barker V.A., Neytcheva M., Polman B.: Solving the Stokes problem on a massively parallel computer. Mathematical Modelling and Analysis, 4 (2000), 1-22.

3. Axelsson O., Gustafsson I.: An iterative solver for a mixed variable variational formulation of the (first) biharmonic problem. Computer methods in Applied Mechanics and Engineering, 20 (1979), 9-16.

4. Axelsson O., Gustafsson I.: An efficient finite element method for nonlinear diffusion problems. Bulletin Greek Mathematical Society, 22 (1991), 45-61.

5. Axelsson O., Makarov M.: On a generalized conjugate orthogonal residual method, Numerical Linear Algebra with Applications, 2 (1995), 467-480.

6. Axelsson O., Vassilevski P.S.: Variable-step multilevel preconditioning methods. I. Selfadjoint and positive definite elliptic problems. Numer. Linear Algebra Appl. 1 (1994), 75-101.

7. Braess D.: Finite elements. Theory, fast solvers, and applications in solid mechanics. Cambridge University Press, Cambridge, 2001. (Second edition)

8. Elman H.C.: Preconditioning for the steady-state Navier-Stokes equations with low viscosity. SIAM Journal on Scientific Computing, 20 (1999), 1299-1316.

9. H.C. Elman and D. Silvester, Fast nonsymmetric iterations and preconditioning for Navier-Stokes equations. SIAM Journal on Scientific Computing, 17 (1996), 33-46.

10. Ewing R.E., Lazarov R., Lu P., Vassilevski P.: Preconditioning indefinite systems arising from mixed finite element discretization of second order elliptic problems. In Axelsson O., Kolotilina L. (eds.): Lecture Notes in Mathematics No. 1457, Springer-Verlag, Berlin, 1990.

11. Luksăn L., Vlček J.: Indefinitely preconditioned inexact Newton method for large sparse equality constrained non-linear programming problems. Numerical Linear Algebra with Applications, 5(1998), 219-247. 